\title{
OBTENCIÓN Y CARACTERIZACIÓN DE CAPSAICINA Y OLEORRESINA DE DOS ESPECIES DE Capsicum, PROVENIENTES DE LA COSTA PERUANA
}

\section{PREPARATION AND CHARACTERIZATION OF CAPSAICIN AND OLEORESIN OF TWO SPECIES OF CAPSICUM, FROM THE PERUVIAN COAST}

\author{
Flor de María Peña Rivera, Narciso Gómez Villanes \\ Laboratorio de Bioquímica y Química de la Facultad de Agronomía - UNCP
}

\section{RESUMEN}

En la presente investigación se llevó a cabo la extracción y caracterización de la oleorresina y capsaicina de Capsicum chinense y Capsicum baccatum, en la Facultad de Agronomía de la Universidad Nacional del Centro del Perú, con el apoyo de otro laboratorio particular de la ciudad de Lima, evaluando el rendimiento y la calidad en función de dos factores: concentración de alcohol etílico de $70 \%$ y $95 \%$, y para el segundo factor 2 niveles fresco y seco con una humedad del 10\%. Los resultados obtenidos de porcentaje de Capsaicina en la oleorresina y porcentaje de capsaicina se obtuvieron mediante el método de Cromatografía Liquida de alta resolución HPLC ISO 7543-2 las cuales se interpretaron utilizando el análisis de varianza, llegando a concluir que: en el rendimiento de oleorresina obtenida, existe efecto significativo en la interacción de la concentración del solvente con el nivel de deshidratación. Los factores estudiados influyen en el porcentaje de capsaicina contenido en la oleorresina, pero de manera individual: comparativamente existen mayores porcentajes de alcaloide para Capsicum chinense frente a Capsicum baccatum en material seco sobre el fresco y para la concentración del alcohol etílico del $95 \%$ sobre el $70 \%$. Después de aislar el alcaloide sin embargo no hay diferencia significativa para el contenido de capsaicina por efecto de los factores estudiados. De esta manera se logró determinar las condiciones para obtener el mejor porcentaje de capsaicina en la oleorresina.

Palabras clave: Capsaicin, Capsicum chinense, Capsicum baccatum, HPLC

\begin{abstract}
In the present investigation was carried out the extraction and characterization of the oleoresin and capsaicin in Capsicum chinense, Capsicum baccatum, in the Faculty of Agronomy,Faculty of the Universidad Nacional del Centro del Peru, with support from other private laboratory in the city of Lima evaluating the performance and quality in terms of two factors: concentration of ethyl alcohol of $70 \%$ and $95 \%$, and for the second factor 2 levels with a cool dry $10 \%$ moisture. The percentage results obtained in the oleoresin capsaicin and capsaicin percentage obtained by the method of high performance liquid chromatography HPLC resolution ISO 7543-2 which is interpreted using the analysis of variance, reaching the conclusion that: in the oleoresin yield obtained, there is significant effect on the interaction of the solvent with the concentration level of dehydration. The studied factors influencing the content in percentage of capsaicin oleoresin but individually: comparatively higher percentages of alkaloid exist for Capsicum chinense against Capsicum baccatum on the dry material to cool and ethyl alcohol concentration of $95 \%$ on $70 \%$. After isolating the alkaloid however no significant difference for the effect of capsaicin content of the factors studied. In this way it was possible to determine the conditions for the best percentage in the oleoresin capsaicin.
\end{abstract}

Keywords: Capsaicin, Capsicum chinense, Capsicum baccatum, HPLC 


\section{INTRODUCCION}

Además del consumo humano, y por los estudios ya realizados se están demandando muchas especies de Capsicum, con las cuales se pueden producir fármacos contra presión alta, reumatismo, varices, artritis, asma, problemas digestivos y como fuente de vitamina C.

Los usos de los frutos naturales o procesados de Capsicum son múltiples. Aparte del consumo en fresco, cocido o como condimento o "especia" en comidas típicas de nuestro país. Además, un uso de significación del ají, es como materia prima para, la obtención de colorantes y de oleorresinas para fines industriales.

Los "picantes" del fruto es variable según el cultivar y el método tradicional de estimarla es la determinación del valor recíproco de la dilución máxima que permite detectar pungencia al gusto el resultado se expresa en unidades Scoville (US).

Según UNCTAD/GATT, aunque la oleorresina de páprika se deriva de un pimiento de la especie capsicum, el término "oleorresina de capsico" denota generalmente las oleorresinas más picantes que habitualmente se extraen de capsicum frutenses y variedades afines. El principio principal del picante, aunque no es el único es la capsaicina química.

La demanda de oleorresina de capsico está aumentando en estos ultimos años . a la larga podría haber buenas perspectivas en el mércado para una mayor producción de los países que actualmente producen especias.

Según Sharapin (1998), la capsaicina se clasifica como un alcaloide. Uno de los métodos de extracción para alcaloides se usó para aislar la capsaicina. Debido a la diversidad y la complejidad de sus estructuras químicas, la nomenclatura de los alcaloides no ha sido esquematizada y su clasificación ha sido realizada por semejanza con estructuras moleculares simples.

El proyecto que a continuación se presenta, trata sobre la obtención y caracterización de la oleorresina y capsaicina, de 2 especies de capsicum, aji mirasol (Capsicum baccatum), y ají panca (Capsicum chinense) cultivadas en el Perú en la Costa Peruana ( Pisco, Cañete, Huaral), ultimamente está siendo cultivada a mayor escala y exportada en forma deshidratada, ya que según consideraciones económicas la utilidad del ají panca y mirasol fresco o deshidratado en polvo en relación con otros cultivos es significativamente superior. Como alternativa de proceso para estos productos no tradicionales se plantea en este proyecto extraer la oleorresina de las dos especies de capsicum lo que le aumenta su valor agregado al ser un producto de fácil transporte, por ser volumenes pequeños y que posee gran estabilidad lo cual hace resistente al ataque de microorganismos y evita su descomposición.

Para tal efecto se realizó un experimento bifactorial en el que se utilizaron dos concentraciones de alcohol etílico al $70 \%$ y al $95 \%$ y 2 niveles de deshidratación en fresco y en seco, utilizando dos especies de capsicum y determinar cuales son las combinaciones de las variables que permitan obtener rendimientos más altos de oleorresina y capsaicina, al mismo tiempo las condiciones apropiadads para la extracción.

\section{MATERIAL Y MÉTODOS \\ Materiales \\ Muestras vegetales \\ Género: Capsicum}

Especie: Capsicum chinense y Capsicum baccatum

Nombre común: ají panca y aji mirasol

Equipos instrumentales de laboratorio

Rotavapor

Mantas de calentamiento

Extractor Soxhlet

Instrumento analítico de Cromatografia liquida de alta Eficiencia (HPLC)

\section{Insumos y reactivos}

Etanol 99\%. Densidad: 0,79 g/ml.Temperatura: 20 ${ }^{\circ} \mathrm{C}$

Cloroformo $90-95 \%$

\section{Materia prima (preparación de muestras)}

La materia prima se adquirió del mercado de Santa Anita de la ciudad se Lima, en estado fresco y seco. Se colocó la materia prima previamente lavadas, en 
bolsas herméticas y después se procedió a secar en un secador de bandejas. Para las extracciones de material seco, este se molió en un molino de discos y luego se tamizó para tener el control de la granulometría del mismo.

Las oleorresinas obtenidas en cada extracción se concentraron y luego fueron pasados en un rotavapor para eliminar el resto del solvente. Este producto fue almacenado en fracios limpios de color ambar, debidamente etiquetados a una temperatura de $5^{\circ} \mathrm{C}$ (refrigerados) para el análisis de cromatografía HLPC.

Seguidamente las muestras de oleorresina fueron tratadas con la metodología para aislamiento de alcaloides, descrito en el libro del Doctor Nicolai Sharapin "Fundamentos de tecnologia de productos Fitoterapéuticos"

El producto obtenido con este proceso fue analizado también con el HPLC pero se presentó la dificultad de la separación de la capsaicina de la fase cloroformica, ya que al evaporar, la capsaicina se evaporaba con el cloroformo y por ello se analizó también a la mezcla clorofórmica

\section{MÉTODO}

\section{A. MÉTODO PARA LA OBTENCIÓN DE LA OLEORRESINA}

La operación para trabajar con materia prima en seco con el $10 \%$ de humedad se realizó secando la muestra, se procedió a pesarla y a reducirla a una malla de 200 mesh. Luego se procesó el material introduciendo en el extractor durante 12 horas en maceración con el solvente. Después se filtró y se llevó a cabo a la destilación al vacío, en el cual se separó el solvente. La oleorresina se llevó al rotavapor para su concentración final. Ello se repitió para todos los experimentos.

\section{B. MÉTODO PARA EL AISLAMIENTO DE LA CAPSAICINA}

Siendo la capsaicina, un alcaloide, según la bibliografía consultada, se procedió según la metodología de extracción de alcaloides a nivel de laboratorio

\section{EXTRACCIÓN DE ALCALOIDES, PROCESO REALIZADO EN EL LABORATORIO}

a. Se inició con la droga vegetal y se extrajo con etanol.

b. Se concentró hasta la consistencia de jarabe, este proceso que en el presente trabajo se le ha denominado extracción de la oleorresina.

c. Se adicionó $\mathrm{H} 2 \mathrm{SO} 4$ hasta $\mathrm{pH} 3,5$

d. Luego se calentó hasta la eliminación del etanol.

e. Se agregó cloroformo y agitó durante 3 horas.

f. Se dejo reposar la muestra toda la noche.

g. Se separó las fases en una ampolla de decantación

h. La fase acuosa ácida se alcalinizó con $\mathrm{NH} 4 \mathrm{OH}$ hasta un $\mathrm{pH}$ 9,5.

i. Se adicionó cloroformo y se agitó durante 1 hora

j. Reposo de la muestra en ampolla de decantación hasta que se separen las fases

k. Se reunieron las fases clorofórmicas de las lavadas, la parte acuosa se deshecho.

I. Se evaporó el solvente de la fase cloroformica, al vacío y a una temperatura de $0^{\circ} \mathrm{C}$ para dejar la base libre.

\section{ANÁLISIS CROMATOGRÁFICO}

Las muestras obtenidas de las 2 especies de capsicum fueron colocadas en viales ámbar de 8 $\mathrm{mL}$. de capacidad. Posteriormente fueron llevadas al laboratorio de análisis instrumental de la ciudad de Lima para el análisis de cromatografía líquida de alta resolución HLPC

\section{E. METODOLOGIA EXPERIMENTAL}

\section{Diseño de tratamientos:}

Para la obtención de la oleorresina de las 2 especies de capsicum estudiadas, se procedió a colocar la materia prima en contacto con el 
solvente, cuya técnica es llamada maceración. Se utilizó alcohol etílico como solvente con 2 concentraciones diferentes, al $70 \%$ y al $95 \%$, la materia prima se trabajó en fresco y en seco con un $10 \%$ de humedad.

En la investigación se utilizó un diseño completamente al azar, en el cual se aplicó un experimento bifactorial los cuales son 2 concentraciones de alcohol y dos niveles de deshidratación (seco y en fresco) utilizando dos especies de capsicum dando como resultado ocho tratamientos con tres repeticiones, cada uno para ser un total de veinticuatro experimentos.

Para el análisis de los resultados se utilizó el modelo estadistíco de análisis de varianza, haciendo uso del programa estadístico Statical Analysis System (SAS) y se comprobó los resultados bajo la prueba de Duncan

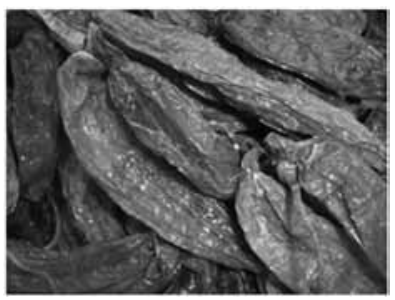

Ají mirasol

Capsicum Baccatum

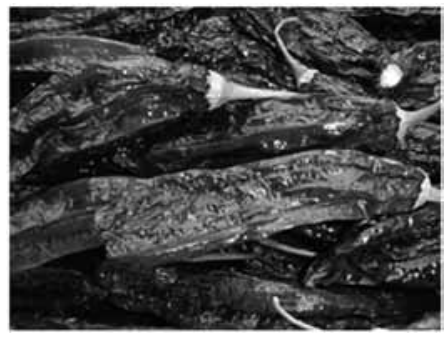

Ají panca

Capsicum Chinense

\section{RESULTADOS}

\section{Rendimiento de oleorresina y capsaicina}

Los siguientes cuadros muestran los rendimientos obtenidos tanto de oleorresina como de capsaicina en las muestras de las dos especies de capsicum estudiadas; así como el porcentaje de capsaicina en las muestra de oleorresinas

Cuadro 1. Rendimiento porcentual de oleorresina en las dos especies de Capsicum, Ají panca (Capsicum chinense) y Ají mirasol (Capsicum baccatum).

\begin{tabular}{|c|c|c|c|c|}
\hline \multirow{3}{*}{ Especies vegetales } & \multicolumn{4}{|c|}{ HUMEDAD } \\
\hline & \multicolumn{2}{|c|}{ Fresco } & \multicolumn{2}{|c|}{ Seco } \\
\hline & Etanol $70 \%$ & Etanol $95 \%$ & Etanol $70 \%$ & Etanol $95 \%$ \\
\hline $\begin{array}{l}\text { aji panca (Capsicum } \\
\text { chinense) }\end{array}$ & 10.4716 & 11.9368 & 14.6221 & 11.1476 \\
\hline $\begin{array}{l}\text { aji mirasol (Capsicum } \\
\text { baccatum) }\end{array}$ & 4.5811 & 3.8846 & 7.3915 & 7.7422 \\
\hline
\end{tabular}

Cuadro 2. Rendimiento porcentual de capsaicina obtenida en las dos especies de Capsicum Ají panca (Capsicum chinense) y Ají mirasol (Capsicum baccatum)

\begin{tabular}{lllll}
\hline & \multicolumn{3}{c}{ HUMEDAD } & \\
\multicolumn{1}{c}{ Especies } & \multicolumn{2}{c}{ Fresco } & \multicolumn{2}{c}{ Seco } \\
vegetales & Etanol 70\% & Etanol 95\% & Etanol 70\% & Etanol 95\% \\
\hline $\begin{array}{l}\text { Aji panca } \\
\text { (Capsicum }\end{array}$ & 0.00071151 & 0.00148233 & 0.00051710 & 0.00116285 \\
chinense) & & & & \\
$\begin{array}{l}\text { Aji mirasol } \\
\text { (Capsicum } \\
\text { baccatum) }\end{array}$ & 0.00062245 & 0.00006070 & 0.00032245 & 0.00195452 \\
\hline
\end{tabular}

Cuadro 3. Porcentaje de capsaicina en la oleorresina obtenida en las dos especies de Capsicum Ají panca (Capsicum chinense) y Ají mirasol (Capsicum baccatum)

\begin{tabular}{|c|c|c|c|c|}
\hline \multirow{3}{*}{ Especies vegetales } & \multicolumn{4}{|c|}{ HUMEDAD } \\
\hline & \multicolumn{2}{|c|}{ Fresco } & \multicolumn{2}{|c|}{ Seco } \\
\hline & Etanol $70 \%$ & Etanol $95 \%$ & Etanol $70 \%$ & Etanol $95 \%$ \\
\hline $\begin{array}{l}\text { Aji panca (Capsicum } \\
\text { chinense) }\end{array}$ & 1.2924 & 1.8673 & 2.9445 & 10.2853 \\
\hline $\begin{array}{l}\text { Ají mirasol (Capsicum } \\
\text { baccatum) }\end{array}$ & 0.4857 & 1.2448 & 1.3237 & 3.0833 \\
\hline
\end{tabular}

\section{RESULTADOS DEL ANÁLISIS DE VARIANZA}

Los siguientes cuadros muestran el análisis estadístico realizado a los resultados obtenidos de rendimiento de oleorresina, rendimiento de capsaicina y porcentaje de capsaicina en la oleorresina utilizando materia prima fresca y seca al $10 \%$ de humedad, con alcohol etílico al $70 \%$ y $95 \%$. 
Cuadro 4. Análisis de variancia para los rendimientos porcentuales de oleorresina en las dos especies de Capsicum Ají panca (Capsicum chinense) y Ají mirasol (Capsicum baccatum)

\begin{tabular}{|c|c|c|c|c|c|}
\hline Fuente & G.L & $\begin{array}{c}\text { Suma } \\
\text { Cuadrados }\end{array}$ & $\begin{array}{c}\text { Media } \\
\text { cuadrados }\end{array}$ & $\begin{array}{c}\text { Valor } \\
\mathrm{F}\end{array}$ & $\operatorname{Pr}>\mathrm{F}$ \\
\hline Especie & 2 & 147.40237566 & 73.70118783 & 11.87 & 0.0018 \\
\hline Estado & 1 & 14.28617412 & 14.28617412 & 2.30 & 0.1576 \\
\hline Solvente & 1 & 16.17353598 & 16.17353598 & 2.60 & 0.1349 \\
\hline Especie Estado & 2 & 13.07584658 & 6.53792329 & 1.05 & 0.3817 \\
\hline Especie Solvente & 2 & 14.17688211 & 7.08844106 & 1.14 & 0.3545 \\
\hline Estado Solvente & 1 & 47.88459750 & 47.88459750 & 7.71 & 0.0180 \\
\hline $\begin{array}{l}\text { Espec. Estado. } \\
\text { Solvente }\end{array}$ & 2 & 50.44986817 & 25.22493409 & 4.06 & 0.0478 \\
\hline $\begin{array}{l}\text { Coeficiente } \\
\text { Determinación } \\
0.819296\end{array}$ & $\begin{array}{l}\text { Coeficien } \\
\text { Variación } \\
28.39145 \\
\end{array}$ & $\begin{array}{ll}\text { nte } & \text { Raiz cuadr } \\
7 & \text { error estan } \\
5 & 2.4921439 \\
\end{array}$ & $\begin{array}{ll}\text { ada } & \text { Porcentaje } \\
\text { dar } & \text { Promedio } \\
& 8.7777958 \\
\end{array}$ & & \\
\hline
\end{tabular}

Cuadro 5. Análisis de variancia para los rendimientos porcentuales de oleorresina en las dos especies de Capsicum ají panca (Capsicum chinense) y ají mirasol (Capsicum baccatum)

\begin{tabular}{|c|c|c|c|c|c|}
\hline Fuente & G.L & $\begin{array}{c}\text { Suma } \\
\text { Cuadrados }\end{array}$ & $\begin{array}{c}\text { Media } \\
\text { cuadrados }\end{array}$ & $\begin{array}{c}\text { Valor } \\
\mathbf{F} \\
\end{array}$ & $\operatorname{Pr}>\mathrm{F}$ \\
\hline Especie & 2 & 0.00000197 & 0.00000099 & 2.52 & 0.1255 \\
\hline Estado & 1 & 0.00000046 & 0.00000046 & 1.19 & 0.2991 \\
\hline Solvente & 1 & 0.00000062 & 0.00000062 & 1.58 & 0.2349 \\
\hline Especie Estado & 2 & 0.00000086 & 0.00000043 & 1.10 & 0.3668 \\
\hline Especie Solvente & 2 & 0.00000059 & 0.00000029 & 0.75 & 0.4956 \\
\hline Estado Solvente & 1 & 0.00000112 & 0.00000112 & 2.86 & 0.1189 \\
\hline $\begin{array}{l}\text { Espec. Estado. } \\
\text { Solvente }\end{array}$ & 2 & 0.00000133 & 0.00000066 & 1.70 & 0.2281 \\
\hline $\begin{array}{l}\text { COEFICIENTE } \\
\text { DETERMINACIÓN } \\
0.630393\end{array}$ & & $\begin{array}{l}\text { OEFICIENTE } \\
\text { ARIACIÓN } \\
01.5242 \\
\end{array}$ & $\begin{array}{l}\text { RAIIZ CUADF } \\
\text { ERROR EST } \\
0.0006255 \\
\end{array}$ & $\begin{array}{l}\text { DA } \\
\text { NDAR }\end{array}$ & $\begin{array}{l}\text { PORCENTAJ } \\
\text { PROMEDIO } \\
0.0006161 \\
\end{array}$ \\
\hline
\end{tabular}

Cuadro 6. Análisis de variancia para el porcentaje de capsaicina en las oleorresinas en las dos especies de Capsicum ají panca (Capsicum chinense) y ají mirasol (Capsicum baccatum)

\begin{tabular}{|c|c|c|c|c|c|}
\hline Fuente & G.L & $\begin{array}{c}\text { Suma } \\
\text { Cuadrados }\end{array}$ & $\begin{array}{c}\text { Media } \\
\text { cuadrados }\end{array}$ & $\begin{array}{c}\text { Valor } \\
\mathrm{F}\end{array}$ & $\operatorname{Pr}>\mathrm{F}$ \\
\hline Especie & 2 & 86.76189631 & 43.38094815 & 5.66 & 0.0075 \\
\hline Estado & 1 & 73.66871079 & 73.66871079 & 9.62 & 0.0039 \\
\hline Solvente & 1 & 34.14201278 & 34.14201278 & 4.46 & 0.0422 \\
\hline Especie Estado & 2 & 39.39513592 & 17.97382826 & 2.57 & 0.0912 \\
\hline $\begin{array}{l}\text { Especie } \\
\text { Solvente }\end{array}$ & 2 & 34.95725350 & 19.69756796 & 2.28 & 0.1175 \\
\hline $\begin{array}{l}\text { Estado } \\
\text { Solvente }\end{array}$ & 1 & 21.07902908 & 17.47862675 & 2.75 & 0.1063 \\
\hline $\begin{array}{l}\text { Espec. Estado. } \\
\text { Solvente }\end{array}$ & 2 & 25.73285860 & 21.07902908 & 1.68 & 0.2015 \\
\hline $\begin{array}{c}\text { COEFICIENTE } \\
\text { DETERMINACIÓ } \\
2.2189062\end{array}$ & & $\begin{array}{c}\text { COEFICIENTE } \\
\text { VARIACIÓN } \\
0.575191\end{array}$ & $\begin{array}{r}\text { RAIIZ CUADR } \\
\text { ERROR ESTÁ } \\
124.7318\end{array}$ & $\begin{array}{l}\text { ADA } \\
\text { NDAR }\end{array}$ & $\begin{array}{c}\text { PORCENTAJE } \\
\text { PROMEDIO } \\
2.7676812 \\
\end{array}$ \\
\hline
\end{tabular}

\section{DISCUSION}

Basándose en el análisis de varianza para el porcentaje de rendimiento de oleorresina se observa que existe diferencia significativa en el factor especie, tanto al $5 \%$ como al $1 \%$ del nivel de significancia. La prueba de Duncan indica que la diferencia hay en las dos muestra vegetales. Así el ají panca (Capsicum chinense) tienen mayor rendimiento $11.9 \%$, mientras que el ají mirasol (Capsicum baccatum) presenta $5.8 \%$. El nivel de deshidratación del ají, fresco y seco con humedad del $10 \%$ y la concentración del solvente, $70 \%$ y $95 \%$, no influyen en el rendimiento de oleorresina pues entre sus niveles no existe diferencia significativa

A nivel de interacciones la única significativa es el nivel de deshidratación - solvente, además de nivel de deshidratación -solvente-especie, esto significa que el nivel de deshidratación, seco 0 fresco en cualquiera de las dos especies influye en el rendimiento de la oleorresina por efecto de la concentración del solvente. El comportamiento que exhibe la variable rendimiento frente a la concentración del solvente está condicionado por el nivel de deshidratación.

Se puede determinar que para los valores promedio de porcentaje de oleorresina en función del solvente utilizado un mayor rendimiento, $14,6221 \%$, utilizando muestras secas y alcohol al $70 \%$. La calidad de oleorresina se evaluó por el porcentaje de capsacina que ésta contenga. Se obtiene un mayor porcentaje de capsaicina en la oleorresina utilizando alcohol etilíco al 95\%, un $10.28 \%$ en muestras de ají panca en fresco y seco.

El análisis de varianza para la variable rendimiento de capsaicina indica que no existe diferencia significativa entre ningún tratamiento, tomando en cuenta que el análisis de varianza de capsaicina en oleorresina indica que los dos factores influyen por separado.

\section{CONCLUSIONES}

- De las dos especies de Capsicum, ají panca (Capsicum chinense) y ají mirasol (Capsicum baccatum), se obtuvo el mayor porcentaje de capsaicina con en Capsicum chinense cuyo valor fue de $10,28 \%$ que está dentro del rango del valor teórico esperado que es entre $10 \%$ a $12 \%$. 
- El más alto porcentaje de rendimiento de oleorresina es de $14.6221 \%$ se obtienen para la especie aií panca seco (Capsicum chinense) utilizando alcohol etílico al $70 \%$.

- El más alto porcentaje de rendimiento de capsaicina, en función del solvente utilizado fue de $0.002 \%$, para la especie ají panca seco (Capsicum chinense) utilizando alcohol etílico al $95 \%$.

- El nivel de deshidratación del Capsicum fresco y seco con humedad al $10 \%$ y la concentración del solvente no influye en el porcentaje de rendimiento de la oleorresina.

- Para obtener el más alto porcentaje de capsaicina en la oleorresina, la variable que produce un efecto más marcado es el nivel de deshidratación, seguido de la especie y concentración del solvente.

\section{RECOMENDACIONES}

- Los análisis de cromatografía líquida de alta resolución (HPLC deben realizarse inmediatamente después de extraída la oleorresina para evaluar los resultados experimentales y detectar rápidamente las posibles fuentes de error.

- El aislamiento de capsaicina también deben realizarse inmediatamente para evitar su descomposición.

- Se debe controlar sistemáticamente la molienda de muestras y el deshidratado porque probablemente estos factores afectan significativamente en el porcentaje de capsaicina de la oleorresina de las muestras.

\section{REFERENCIAS BIBLIOGRÁFICAS}

1. Ch. C. Composición de los alimentos Peruanos Instituto de Nutrición tercera edición.Lima-Perú, 1962.

2. Chiappe, L. Estudio comparativo de diversas variedades de ají Tesis de Grado LM., 1960.

3. Corrales, N. El cultivo del Ají en el Perú Estación Experimental de Agricultura. La Molina, 1961.

4. Delgado, D. F. Cultivos Hortícolas datos básicos Edi-Agraria Universidad, 1988.

5. ITINTEC Norma técnica: 209.238-1986 Salsa de Ají, 209.035-1970. Pimentón, 209.0701970 Vinagre, 209.132-1986 Determinación de Ceniza, 209.102-1975 "Especies y Condimentos", 203.071-1975 "Productos elaborados a partir de frutas y otros vegetales.

6. Orbegoso, A. Reseña del cultivo, identificación botánica y comparativo de rendimiento en fresco de variedades de ají. Escuela Nacional de Agricultura. La Molina, 1954.

7. Ortiz, R. Utilización de descriptores en la caracterización de líneas de Capsicum. UNA,1983.

8. Velasco, F. Recolección y descripción de muestras del G. Capsicum en la provincia de Satipo (Junin) y San Miguel (Cajamarca).UNA, 1971.

9. Sharapin Nikolai. Fundamentos de tecnología de productos fitoterepauticos. Programa Iberoamericano de Ciencia y Tecnología para el Desarrollo, CYTED, 1998. 